\title{
La accesibilidad alimentaria en la prensa digital: encuadres $y$ representación del hambre en España
}

\section{Food accessibility on digital press: framing and representation of hunger in Spain}

Flora Marín-Murillo. Universidad del País Vasco/EuskalHerrikoUnibertsitatea (UPV/EHU).

España.

flora.marin@ehu.eus

$[\underline{\mathrm{CV}}]$ (C)

José Ignacio Armentia-Vizuete. Universidad del País Vasco/EuskalHerrikoUnibertsitatea

(UPV/EHU). España.

ignacio.armentia@,ehu.eus

$[\underline{\mathrm{CV}}]$ (1) $\mathrm{G}$

Iñigo Marauri-Castillo. Universidad del País Vasco/EuskalHerrikoUnibertsitatea (UPV/EHU).

España.

inigo.marauri@ehu.es

$[\underline{\mathrm{CV}}]$ (c) $\mathrm{G}$

María del Mar Rodríguez-González. Universidad del País Vasco/EuskalHerrikoUnibertsitatea (UPV/EHU). España.

marialdemar.rodriguez@,ehu.es

$[\underline{\mathrm{CV}}]$ (1) $\mathrm{C}$

Este artículo es parte del proyecto "Seguridad Alimentaria y Cibermedios: Temáticas, nuevas fuentes y servicios". Este proyecto, con referencia CSO2017-82853-R, ha obtenido financiación en la convocatoria 2017 de los proyectos de $I+D+I$ del programa estatal de Investigación, Desarrollo e Innovación orientada a los retos de la sociedad promovido por el Ministerio de Economía, Industria y Competitividad. Sus autores forman parte del Grupo de Investigación MediaIker (referencia GIU16/08).

Cómo citar este artículo / Referencia normalizada

Marín-Murillo, F., Armentia-Vizuete, J. L., Marauri-Castillo, I y Rodríguez-González, M. N. (2020). La accesibilidad alimentaria en la prensa digital: encuadres y representación del hambre en España. Revista Latina de Comunicación Social, 75, 169-187.

https://www.doi.org/10.4185/RLCS-2020-1421

\section{RESUMEN}

Introducción: El objetivo principal de este trabajo es conocer la relevancia que los diarios digitales españoles dan a las dificultades de una parte de la población española para acceder a una alimentación suficiente. Se toman en cuenta para ello conceptos como hambre oculta y accesibilidad alimentaria. Metodología: Se han analizado los contenidos publicados en 2017 por cuatro cabeceras digitales (elpais.com, lavanguardia.com, abc.es y eldiario.es) por medio del análisis de contenido y la teoría del framing. Resultados y conclusiones: De esta investigación se desprende la complejidad del tema y la diversidad de encuadres: Solidaridad, Hambre/Hambruna, Despilfarro y Acceso. 
Asimismo, se constata una traslación del ámbito internacional al nacional. El hambre en España es un "hambre oculta" y se construye como un problema que afecta a individuos "desfavorecidos", sin rostro, donde las causas se eluden generalmente y las soluciones son monopolizadas por las iniciativas solidarias.

PALABRAS CLAVE: hambre; encuadre; accesibilidad alimentaria; prensa digital.

\begin{abstract}
Introduction: The main objective of this paper is to analyse the relevance that Spanish digital newspapers grant to the struggles of a part of the Spanish population to access a sufficient nutrition. Concepts like hidden hunger and food accessibility are used to understand this. Methodology: the contents published in 2017 by our digital mastheads (elpais.com, lavanguardia.com, abc.es and eldiario.es) were analysed by means of content analysis and the framing theory. Results and conclusions: The findings of this study disclose the complexity of the subject and the diversity of framings: Solidarity, Hunger/ Famine, Waste and Access. Likewise, a translation from the international to the national scope is confirmed. The hunger in Spain is a "hidden hunger" and it is constructed as a problem that impacts "disadvantaged" individuals, faceless, where causes are generally avoided, and the solutions are monopolised by supportive initiatives.
\end{abstract}

KEYWORDS: hunger; framing; food accessibility; digital press.

\title{
CONTENIDO
}

1. Introducción. 2. Objetivos e hipótesis. 3. Metodología. 4. Resultados. 4.1. Relevancia de los temas de alimentación. 4.2. Accesibilidad: encuadres temáticos y ámbito geográfico. 4.3. La construcción mediática del "hambre local". 5. Discusión y conclusiones. 6. Referencias bibliográficas.

Artículo traducido por Yuhanny Henares (Traductora académica, Universitat de Barcelona).

\section{Introducción}

Los problemas para obtener alimentos se relacionan con países pobres o en vías de desarrollo. Sin embargo, la dificultad para garantizar el acceso a alimentos, en particular los productos más saludables, también es una realidad de los países más industrializados o desarrollados.

El periodista y escritor argentino Martín Caparrós escribe en su obra El Hambre que el concepto que da título a su libro es una metáfora de muchas cosas y entre ellas de la división: "Una barrera tajante entre ellos y nosotros, los que tienen y los que no tienen (...)" (Caparrós, 2014, p. 520). Esa barrera, que no es sólo simbólica sino en muchos casos es también física y se levanta entre países, como Nigeria, Sudán o Afganistán, está empezando a desdibujarse. De la crisis económica, de la precariedad, de las políticas neoliberales, de la burbuja inmobiliaria, de los recortes en ayudas sociales, entre otros factores, ha prendido en los llamados países desarrollados lo que la FAO describe en su Portal Terminológico como "hambre oculta" o "hambre encubierta". Por esta modalidad de hambre "se entiende la carencia sostenida de aporte de vitaminas y minerales en relación con las necesidades de una persona. Las carencias más prevalentes a nivel mundial son las de hierro, yodo y vitamina A" (http://www.fao.org/faoterm/es/?defaultCollId=6). Se calcula que hay 2.000 millones de personas en el mundo con carencia de hierro, en su gran mayoría mujeres y niños. Unos 1.500 millones tienen deficiencia de yodo y 800 millones de vitamina A. Incluso cuando los aportes energéticos sean los adecuados estas insuficiencias pueden existir. El calificativo de oculta o encubierta se debe a que en muchos casos, cuando se trata de cuadros leves, no se aprecian signos visibles; e incluso cuando la situación se agrava los pacientes no son muy conscientes de ello, ya que no suelen disponer de la suficiente información para detectar los síntomas físicos que se vinculan a ella. 
La obesidad no sólo no está reñida con el hambre oculta. Cada vez más las personas con sobrepeso en los países desarrollados sufren una ingesta excesiva de calorías y una manifiesta escasez de nutrientes. En definitiva, están malnutridos y son víctimas de este "hambre oculta".

El problema del hambre no es ya que no se produzcan suficientes alimentos en el mundo, sino más bien las dificultades que cada vez una mayor parte de la población tiene para acceder a ellos. De ahí que exista una estrecha relación entre los conceptos de hambre y de accesibilidad alimentaria. "Sin resolver esta cuestión, la mayor pandemia no contagiosa que sufre la humanidad seguirá creciendo y, en consecuencia, sin lograr alcanzar la seguridad alimentaria ponemos en cuestión la paz y la seguridad en el mundo" (Castro, 2013, p. 12).

La alimentación es un derecho que ya fue recogido en el artículo 25 en la Declaración de los Derechos Humanos, y desde entonces ha sido reformulado y adaptado a través de compromisos y pactos internacionales.

En el año 2013 el entonces relator de la ONU, Jean Ziegler, lo definió como

el derecho a tener acceso, de manera regular, permanente y libre, directamente o mediante compra con dinero, a una alimentación cuantitativa y cualitativamente adecuada y suficiente, que corresponda a las tradiciones culturales de la población a que pertenece el consumidor y que garantice una vida psíquica y física, individual y colectiva, libre de angustias, satisfactoria y digna. (Ziegler, 2003)

Ante esta compleja realidad los medios de comunicación tienen una responsabilidad innegable. La información sobre seguridad alimentaria, y por ende, sobre la alimentación, la nutrición, gastronomía, industria alimentaria, etc. ha incrementado su presencia en los medios de comunicación de forma notable. Los diferentes informes realizados en torno a esta temática en España así nos lo indican (Elika, 2013; 2014; 2015; 2016).

En 1996 la Organización de las Naciones Unidas para la Alimentación y Agricultura, FAO, definió el concepto de Seguridad Alimentaria "Food Security" como aquella situación "en que todas las personas tienen en todo momento acceso físico, social y económico a alimentos suficientes, inocuos y nutritivos, que satisfacen sus necesidades energéticas diarias y preferentemente alimentarias para llevar una vida sana y activa" (FAO, 1996). Es preciso matizar que este concepto es diferente a "Food Safety", que se circunscribe a la inocuidad de los alimentos, a su salubridad, y que es la acepción a la que se asocia con más frecuencia en castellano la locución Seguridad Alimentaria.

Del conjunto de temas sobre Alimentación que han sido y son objeto de estudio, la accesibilidad como concepto que abarca un compendio de realidades -la carestía de los alimentos, el despilfarro, la escasez o las dificultades para acceder a alimentos- ha merecido una atención desigual desde el punto de vista comunicacional.

El aspecto más extremo vinculado con la accesibilidad es el hambre. Se trata de un término subjetivo y mediático que la FAO define como "escasez de alimentos básicos que causa carestía y miseria generalizada". Mientras, la hambruna es "el resultado de una secuencia de procesos y sucesos que reduce la disponibilidad de alimentos o el derecho al alimento, causando un aumento notable y propagado de la morbilidad y mortalidad". (FAO, http://www.fao.org/3/a-at772s.pdf). La hambruna 
se asocia a los conflictos y las catástrofes naturales, habitualmente sequías, y las soluciones se orientan en la actualidad a la aplicación de políticas y programas junto a la movilización de recursos suficientes.

Los reportajes que la revista Life publicó en julio de 1968 sobre Biafra y el que Michael Buerk de la BBC editó sobre el "hambre bíblica" de Etiopía, en octubre de 1984, señalan el origen de la hambruna como fenómeno mediático. Como expresión más extrema de la accesibilidad, el tratamiento informativo del hambre es analizado dentro y fuera de nuestras fronteras por diversos autores/as y desde distintas perspectivas. Así, en el entorno internacional podemos destacar el trabajo de Odesanya et al. (2015) sobre la cobertura que The Guardian hace de la Seguridad alimentaria en Nigeria, o la aportación del tratamiento mediático de los medios de comunicación americanos sobre el hambre en el Cuerno de África (Sorenson, 1991). A nivel visual, la construcción de la imagen del hambre es tratada por Clark (2004) mediante el análisis de las polémicas imágenes tomadas por MekanicPhilipos de un bebé sufriendo malnutrición en el centro terapéutico de Yirba cerca de Aswan.

Desde la universidad española cabe apuntar la valiosa aportación de Susana Morais (2015) sobre la representación del hambre en El País y The New York Times.

Mucho menos frecuentes son los trabajos que versan sobre el tratamiento mediático del "hambre oculta" en España. De ahí, el interés del capítulo que Gracia-Arnaiz y García-Oliva (2017) dedican a las retóricas del hambre at home en la prensa digital española, en el que enclavan la aparición de los primeros artículos sobre el tema en 2009, iniciando una tendencia que se vuelve significativa durante 2012-2013, según las autoras (2017, p. 170).

\section{Objetivos e hipótesis}

Esta investigación intenta por tanto llenar el vacío existente sobre la presencia y el tratamiento de la accesibilidad alimentaria en los principales medios de comunicación españoles.

Los objetivos que se plantean son los siguientes:

O1. Establecer el lugar que ocupa la accesibilidad respecto a los contenidos publicados sobre alimentación en los diarios digitales.

O2. Determinar cuáles son los encuadres temáticos sobre accesibilidad alimentaria tratados por estos medios y su relevancia.

O3. Conocer el ámbito geográfico en el que se contextualizan las informaciones sobre accesibilidad.

O4. Analizar en el ámbito nacional la jerarquización de los encuadres sobre accesibilidad y sus posibles consecuencias.

O5. Identificar la presencia explícita e implícita del "hambre local" en los contenidos publicados y su tratamiento informativo.

De la consecución de los objetivos propuestos se pretenden corroborar o refutar las siguientes hipótesis:

H1. Las informaciones relacionadas con la accesibilidad, "foodsecurity”, tienen un peso significativo en la agenda temática. 
H2. Entre los encuadres temáticos más trabajados, los que se asocian con Hambre y con Solidaridad son los más destacados.

H3. El foco de interés sobre el hambre en el mundo se ha trasladado desde el ámbito internacional a lo local.

H4. Las causas y protagonistas del "hambre local" o "hambre oculta" se invisibilizan en los medios estudiados.

H5. La caridad como solución a los problemas derivados por el "hambre local" o "hambre oculta" se fomenta desde los medios de comunicación.

\section{Metodología}

La prensa digital es el medio elegido para la realización de este estudio. Los datos de difusión avalan la expansión de las versiones digitales de los diarios convencionales y de los periódicos nativos frente a la menguante oferta en papel. Según los informes anuales de Asociación de Editores de Diarios de España (AEDE), la prensa española perdió 1.561 .803 ejemplares, el 38\% de su difusión, en el periodo 2001-2013 (2016, p. 81).

Esta tendencia a la baja se mantiene en la actualidad. Según datos de OJD (www.ojd.es), en lo que llevamos de siglo la difusión total de la prensa española ha pasado de 4.107 .959 copias a 1.865.583, una pérdida del $54 \%$.

Los diarios elegidos para el estudio son un nativo digital, eldiario.es, y las versiones digitales de tres periódicos impresos: abc.es, lavanguardia.com y elpais.com. Los cuatro figuraban entre los diez diarios digitales españoles con mayor audiencia en noviembre de 2017, según ComScore, la empresa encargada por la Asociación para la Investigación de los Medios de Comunicación (AIMC) de realizar las mediciones de consumo de información de los diferentes medios digitales.

La selección del objeto de análisis abarca desde el 1 de enero hasta el 31 de diciembre de 2017, y recoge todas aquellas informaciones publicadas durante este periodo que guardan relación con la accesibilidad alimentaria. Para acometer esta tarea se han utilizado los buscadores de los propios medios y empleado las siguientes palabras de búsqueda: agricultura, alimentos, alimentación, alergia, dieta, ganadería, gastronomía, hambre, industria alimentaria, nutrición, obeso, obesidad, pesca, y seguridad alimentaria.

El análisis de contenido ha sido la herramienta metodológica seleccionada. La razón, tal y como defienden autores como Walitzer y Wienir (1978); Krippendorf (1980); Kerlinger (1986) o Bardin (1996), es que permite a quien investiga la sistematización y cuantificación de los contenidos, en el presente caso sobre Accesibilidad, y su contextualización dentro de un marco más genérico como es el de la Alimentación.

Los encuadres que corresponden al ámbito de la accesibilidad han sido inducidos a partir de la propia definición que la FAO (1996) ofrece y la lectura de los contenidos seleccionados. Esta lectura está guiada por las herramientas y dispositivos propuestos por autores como Entman (1993), Gamsom y Modigliani, (1989), y Tankard (2001) para la definición de los marcos noticiosos, atendiendo sobre todo a la inclusión de palabras clave en los titulares, reconocimiento de los protagonistas, las fuentes y los argumentos. 
A partir de ahí se ha diseñado una ficha donde se consignan los encuadres a los que pertenece cada uno de los contenidos, junto al ámbito geográfico al que se alude en la información: nacional, internacional o híbrido.

Una vez excluidos los contenidos de carácter internacional e híbrido se procede a realizar un análisis de los discursos de ámbito nacional. No se trata solo de identificar los marcos noticiosos de la información, sino de establecer un análisis que cuantifique y a la vez describa la identificación de las personas afectadas, la propuesta de soluciones y la exposición de las causas atendiendo a los elementos antes descritos.

\section{Resultados}

\subsection{Relevancia de los temas sobre alimentación}

Durante el 2017 se publicaron en los cuatro diarios un total de 4.517 contenidos relacionados con la alimentación. La distribución es un tanto desigual, pues mientras en abc.es se alcanzan las 2023 informaciones en elpais.com se cifran en 466. Por encima de este último se encuentran lavanguardia.com, que ofrece 753 contenidos, y eldiario.es con 1.275. La abundancia de contenidos en abc.es se debe en gran medida a la inserción de informaciones pertenecientes a otros diarios provinciales y regionales del grupo Vocento.

Los temas sobre alimentación están presentes de forma constante y con pocas variaciones durante todos los meses estudiados. De hecho, las cifras globales oscilan desde los 361 contenidos de enero a los 429 de mayo.

En cuanto a las temáticas, la accesibilidad, como se puede observar en el gráfico 1, protagoniza uno de cada diez contenidos publicados sobre alimentación en los principales diarios digitales españoles, por encima de Seguridad y Riesgo Alimentarios (8\%), y por debajo de Industria Alimentaria (15\%), Agricultura, Ganadería y Pesca (19\%), Nutrición (23\%) y Gastronomía (25\%).

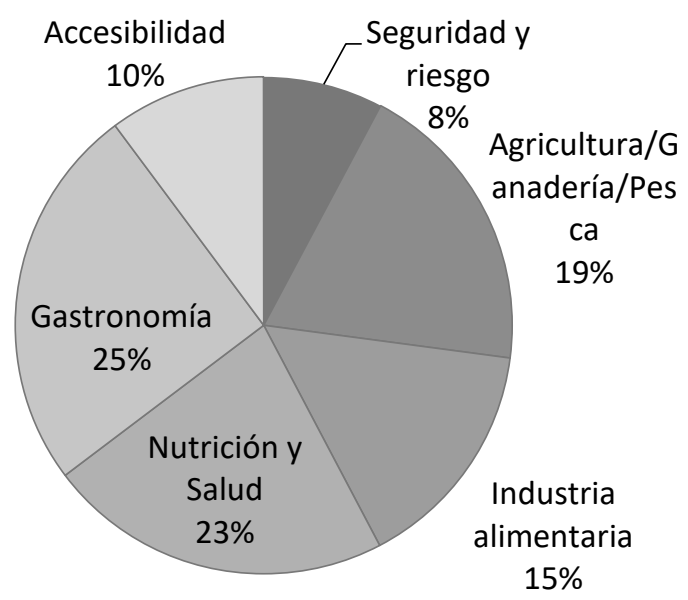

Gráfico 1: distribución por temáticas (\%).

Fuente: elaboración propia.

Aunque hay visibles oscilaciones entre unos meses y otros se constata la publicación constante de este tipo de contenidos durante 2017. Entre los medios estudiados las variaciones son mínimas, al 
menos, entre tres de los cuatro diarios. Abc.es y lavanguardia.com publican 133 contenidos cada uno, mientras eldiario.es llega a 120. La mayor diferencia se halla en elpais.com, cuyo número de contenidos se queda en 74 .

Estas diferencias se invierten si se toma como referencia la proporción de este tipo de contenidos frente al cómputo total de cada diario. Así, quien se queda descolgado en este caso es abc.es, cuyo porcentaje de contenidos sobre accesibilidad respecto al total es de un 6,5\%. En eldiario.es, la proporción asciende al 9,5\%, mientras que en lavanguardia.com sube al 17,7\%, casi dos puntos porcentuales más que en elpais.com $(16 \%)$.

A falta de un estudio diacrónico más extenso podemos afirmar, teniendo en cuenta otras investigaciones, que los textos relacionados con la accesibilidad han incrementado su presencia tanto en la prensa online como la convencional en los últimos años. Así, en el 2012 el Informe Elika referente a la aparición de los contenidos sobre seguridad alimentaria en 9 diarios publicados en el País Vasco o con edición en esta autonomía, situaba la accesibilidad en el tercer puesto de temas más recurrentes, con 132 artículos. Los autores apuntaban a la crisis económica como la causa directa de este incremento: "La crisis económica, que dificulta el acceso de sectores de la población a los alimentos, ha colocado la accesibilidad en cotas elevadas" (Elika, 2012).

En referencia a la prensa digital el trabajo de Gracia y García, citado anteriormente, señala un incremento paulatino de los contenidos sobre la incidencia de la crisis en la alimentación a partir de 2009: "El análisis cronológico indica que, antes de 2009, apenas hay referencias, empiezan a crecer a finales de ese año e inicios de 2010, y pasan a tener una presencia significativa desde 2012-13” (2009, p. 170).

Tabla 1. Distribución de contenidos sobre accesibilidad alimentaria por meses.

\begin{tabular}{|l|l|l|l|l|l|l|l|l|l|l|l|l|l|}
\hline Accesibilidad & ene & feb & mar & abr & may & jun & jul & ago & sep & oct & nov & dic & TOTAL \\
\hline abc.es & 7 & 10 & 13 & 12 & 8 & 12 & 11 & 11 & 10 & 8 & 12 & 19 & 133 \\
\hline eldiario.es & 4 & 7 & 7 & 6 & 15 & 16 & 4 & 9 & 8 & 17 & 9 & 18 & 120 \\
\hline elpais.com & 6 & 8 & 2 & 4 & 4 & 7 & 8 & 4 & 5 & 11 & 9 & 6 & 74 \\
\hline lavanguardia.com & 6 & 8 & 6 & 5 & 8 & 14 & 8 & 5 & 9 & 23 & 11 & 30 & 133 \\
\hline TOTAL & 23 & 33 & 28 & 27 & 35 & 49 & 31 & 29 & 32 & 59 & 41 & 73 & 460 \\
\hline
\end{tabular}

Fuente: elaboración propia.

\subsection{Accesibilidad: encuadres temáticos y ámbito geográfico}

El acceso físico y económico a los alimentos se refleja en los medios de comunicación a través de diferentes informaciones que aluden tanto a las consecuencias derivadas de los impedimentos para conseguir una alimentación adecuada como de las soluciones propuestas para facilitar ese mismo aprovisionamiento. 


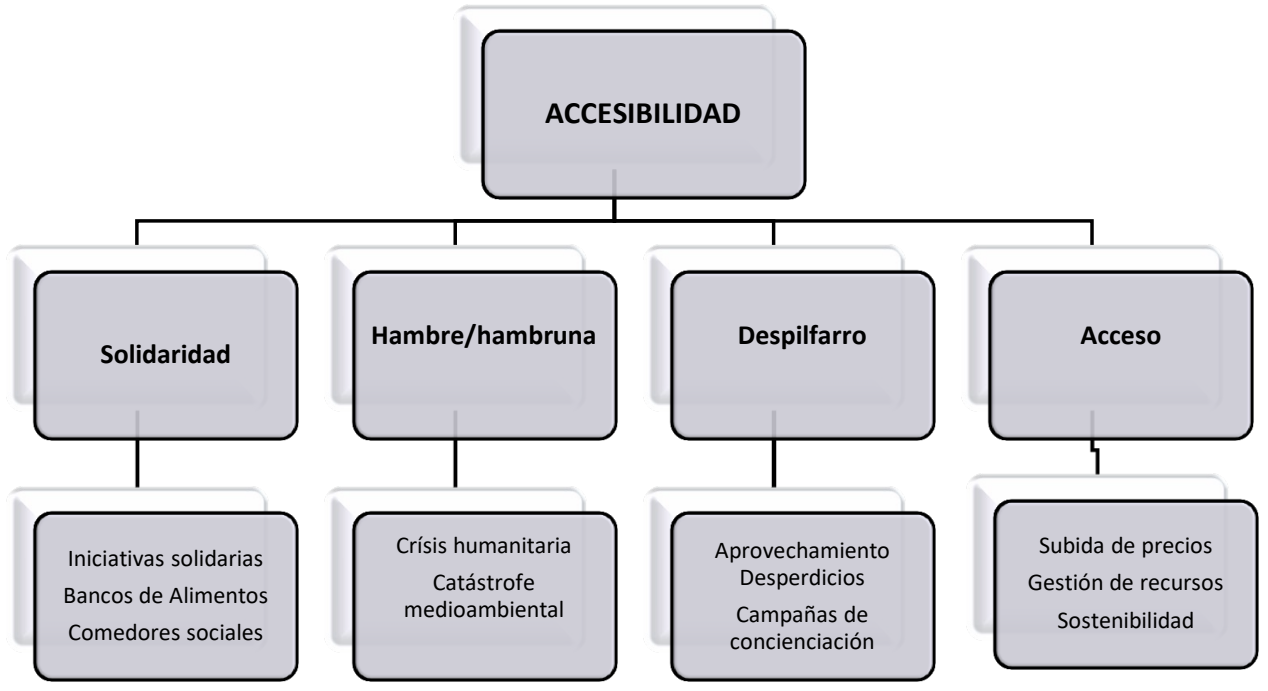

Gráfico 2: encuadres sobre accesibilidad.

Fuente: elaboración propia.

De esta variedad de perspectivas se han inducido cuatro encuadres que cubren la categoría Accesibilidad y su cobertura informativa. La Solidaridad es uno de ellos. Ante la imposibilidad de cubrir las necesidades alimentarias de la población, los bancos de alimentos, los comedores sociales y todo tipo de iniciativas solidarias públicas o privadas son noticia en los medios de comunicación. Este marco, el más recurrente, aparece en el 45\% de las informaciones sobre accesibilidad. En el mes de diciembre las campañas de recogida de alimentos se intensifican y esto hace que sea el periodo con más contenidos.

Entidades bancarias, supermercados o industria alimentaria copan a menudo los titulares como protagonistas de acciones solidarias: “¿Qué hace Mercadona con la comida que no vende?” (abc.es, 04/12/2017); "Danone y Banco de Alimentos de Madrid vuelven a unirse en la iniciativa solidaria 'Maletas contra el hambre" (lavanguardia.com, 08/06/2017). Esta controvertida simbiosis entre mercados y ayuda social da lugar a interesantes reflexiones y conceptos: "Brand Aid" deRichey y Ponte, (2011), el "filantrocapitalismo" de Edwards, (2010), o el "Marketing de los valores" de Lipovetsky (2000).

La gran mayoría de los textos son noticias de actualidad reciente y apenas si se contabilizan artículos de opinión.

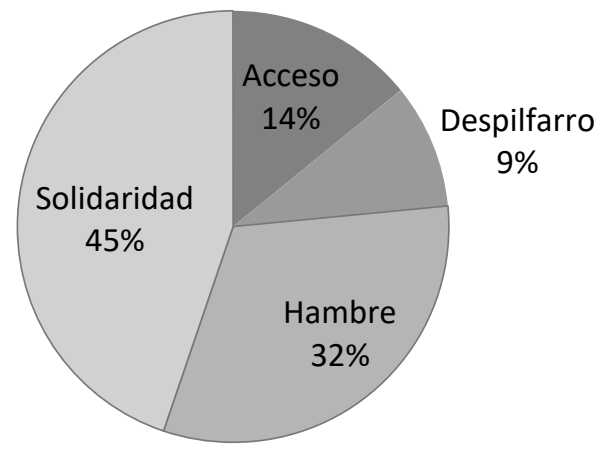

Gráfico 3: \% Encuadres Temáticos.

Fuente: elaboración propia. 
El hambre y/o la hambruna es un problema que afecta prioritariamente al continente africano y en menor medida a Asia y Sudamérica. El 32\% de los textos inciden en este encuadre. El tono de estos contenidos es de denuncia y se prioriza la dimensión política, pero sin olvidar la social y económica. Las crisis humanitarias en determinadas regiones como Somalia, Nigeria y, para abc.es, Venezuela generan un goteo continuo de noticias en las que se analiza y denuncia la situación de riesgo que afecta a la población civil calificándola de "hambruna".

Para elpais.com el hambre es el encuadre de mayor importancia durante el 2017 con un 69\% de contenidos, muy alejado del $29 \%$ de abc.es, $28 \%$ de eldiario.es y $17 \%$ de lavanguardia.com., cabeceras que priorizan el encuadre de Solidaridad.

Morais (2015) en su tesis sobre la representación del hambre en la prensa de referencia entiende que "al privilegiar la etapa Crisis, se destacan más los factores de orden coyuntural y los de carácter estructural ocupan apenas un segundo plano que contrasta con el papel determinante que los estudios hoy disponibles sobre el hambre reiteradamente destacan” (2015, p. 365). Sin embargo, si observamos el encuadre del Hambre/hambruna en su conjunto, se constata que va más allá de la actualidad inmediata para hacerse eco de opiniones e informes de mayor calado. Los artículos de opinión y colaboraciones son más frecuentes en este apartado y generalmente están firmados por activistas de ONG o directivos y colaboradores de instituciones internacionales como la FAO. No hay duda si se observan estos medios que el hambre, asociada a la soberanía alimentaria, la violencia y la preocupación por el cambio climático como elementos desencadenantes de algunas de estas trágicas situaciones, se ha ido asentando en la agenda mediática. El acceso a la alimentación concebido como un derecho fundamental del hombre da lugar también a varias reflexiones.

Otro de los encuadres asociados a la accesibilidad es el Despilfarro. No es casual su relevancia en España, el séptimo país de Europa que más comida desperdicia, en concreto 7,7 millones de toneladas. En términos individuales supondría unos 63 kilos al año por habitante. De ahí, la importancia del aprovechamiento de los excedentes y desperdicios y la toma de medidas para evitar el despilfarro: "Change.org lleva 7 años cambiando (gracias a ti) el mundo" (elpais.com, 22/11/2017), "Nevera solidaria: objetivo, no tirar comida" (lavanguardia.com, 06/01/2017).

Pese a que periódicamente se publican informaciones denunciando el despilfarro y mal uso de los recursos con una clara vocación de concienciación social este encuadre es el menos destacado, con un escaso $9 \%$ de contenidos.

El último encuadre ha sido denominado Acceso. Si se prevé que la población ascienda a unos 10.000 millones para 2050, el problema que se plantea es cómo satisfacer sus necesidades nutricionales. Para alimentar a 2.000 millones de personas más, la producción de alimentos tendrá que aumentar en un $50 \%$ a nivel mundial. Los textos que denuncian esta situación o que informan sobre investigaciones o acciones encaminadas a una gestión inteligente de los recursos tienen cabida en esta categoría: "Más gente, más comida, ¿peor agua?" (elpais.com, 30/08/2017); "Seremos demasiados para el planeta? 11.200 millones de personas en el años 2100" (abc.es, 12/07/2017).

También en este encuadre se hace referencia directa a la carestía, como falta o escasez de algo, por extensión de víveres, y a la subida de sus precios, casi siempre motivados por la incidencia de determinados accidentes climáticos: "El precio de las verduras se dispara por el temporal de frío" (lavanguardia.com, 24/01/2017); "Portugal plantea cortes de agua nocturnos por la grave sequía" (abc.es, 12/07/2017). Otra vertiente del Acceso pone el acento en la accesibilidad favorecida o perjudicada por decisiones de tipo legal, como deducciones fiscales: "Defensor del Pueblo pide deducciones fiscales que compensen gasto de celíacos" (lavanguardia.com, 27/04/2017). 
Estos contenidos, si bien no son los más destacados, ocupan el 14\% de las informaciones, y tienen una presencia diversa en cada uno de los medios analizados: del 18 y 19\% de abc.es y el país.com al 10 y $11 \%$ de lavanguardia.com y eldiario.es.

\section{3. Ámbito geográfico}

Durante muchos años la accesibilidad se ha relacionado con el hambre en el llamado Tercer Mundo, causada por los desplazamientos masivos originados por conflictos violentos y en ocasiones tribales, las sequías o los desastres naturales. Sin embargo, hoy su ámbito de influencia ha llegado a los países desarrollados en forma de malnutrición y obesidad.

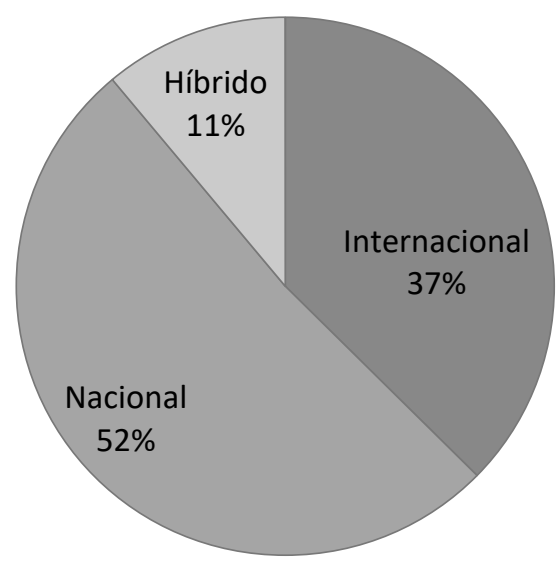

Gráfico 4: accesibilidad: Ámbito geográfico.

Fuente: elaboración propia.

En el 37\% de los textos el ámbito geográfico al que hace referencia la información es internacional, situado prioritariamente en Asia o África, y en menor medida en países del Caribe. Este porcentaje se dobla en elpais.com (73\%), es ligeramente superior en abc.es (39\%) y eldiario.es (34\%); y desciende en el caso de lavanguardia.com a un 19\%. En el caso de elpais.com, más del 50\% están situadas en el continente africano y se centran en la región de la cuenca del lago Chad. Diferentes colaboradores, como JoseGraziano da Silva, director general de la Organización de las Naciones Unidas para la Alimentación y la Agricultura -FAO-, o Enrique Yeves, director de Comunicación de dicha organización, entre otros, reflexionan sobre la hambruna en este territorio, sus causas, consecuencias y medidas para paliarla.

Abc.es se decanta por América del Sur y se fija en Venezuela. De ahí proceden el $50 \%$ de sus informaciones internacionales, que llevan la firma de LudmilaVinogradoff, corresponsal en este país. En estas noticias se alude tanto a la escasez de medicamentos como de alimentos, y van desde la crónica de sucesos -"Aumentan los asesinatos por culpa del hambre en Venezuela" (03/01/207), "Un guardia nacional mata a una mujer embarazada durante reparto de jamones en Venezuela" (31/12/2017)- hasta las numerosas protestas con motivo de la escasez y las restricciones: "Las protestas por falta de comida, agua y gas se extienden por Venezuela", (29/12/2017). Venezuela y Accesibilidad es un binomio recurrente durante todo el 2017 en este medio.

Las informaciones calificadas como híbridas son aquellas que se inscriben en un marco geográfico compartido entre España y Europa o el resto del mundo y suponen un $11 \%$ de los contenidos. 
En términos globales hay que destacar el alcance de la información nacional en esta temática con una implantación del 52\%, 237 contenidos. Estos se reparten de forma bastante desigual por subtemas. Así, destacan las informaciones que hacen referencia a la Solidaridad con un 78\% (184), seguidas de Despilfarro con un 11\% (26), y Acceso con un 9\% (21). Mucho menos frecuentes son las noticias relacionadas de manera explícita con el hambre, donde sólo se contabilizan 6 , es decir un exiguo $2 \%$. Este porcentaje contrasta con el $62 \%$ que sería la presencia de este subtema en un marco internacional.

\subsection{La construcción mediática del "hambre local"}

\section{En España según el Informe sobre el Estado Social de la Nación 2017}

en un $2,6 \%$ de los hogares no puede permitirse comer carne, pollo o pescado al menos cada dos días: son casi medio millón de hogares (477.000) donde no se puede garantizar una alimentación adecuada; en estos hogares viven más de un millón de personas (1,2 millones), entre ellas niños y niñas. Aunque hay estudios que multiplican por cuatro estas cifras oficiales, y estiman que son un $12,4 \%$ los hogares donde no pueden llevar una dieta adecuada, y en un 13,8\% los que no pueden realizar una comida de carne, pollo o pescado al menos tres veces por semana. $(2017$, p. 6$)$

Estas personas sufren "hambre oculta", pero al mismo tiempo se constata que esa escasez de recursos alimentarios, quiénes y por qué la padecen, también es invisible para la sociedad en general. En España el hambre está por tanto doblemente oculta.

A pesar de que sólo en 6 de los 237 textos enmarcados dentro de ámbito nacional la temática central es el hambre, este término aparece en el titular en 11 ocasiones; si bien únicamente en uno de ellos se hace referencia explícita al hambre oculta: "Las entidades sociales alertan de la existencia de "hambre oculta" agravada por la crisis económica" (eldiario.es, 26/05/2017). El artículo cita el informe Fam oculta a Catalunya, un obstacle en la igualtatd'oportunitats (mayo, 2017) donde ponen de manifiesto "una reiterada i suficient manca, en una part significativa delsenquestats, delsmicronutrientsmésinvolucrats en elssímptomesclínics de la fam oculta (vitamines A, ferro i zinc), a mésd’altres” (2017, p. 19).

Las personas afectadas por la inseguridad alimentaria son identificadas en el titular en el 18\% (42) de las informaciones. Son colectivos y sobre todo familias, a las que se califica de "vulnerables", en "riesgo de exclusión", "desfavorecidas", "necesitadas" o simplemente "pobres", con los niños o menores como el grupo con mayor número de menciones. No hay que olvidar que en el Informe UNICEF de abril de 2017, Children of Austerity: Impact of the Great Recessiononchildpoverty in richcountries, se dice que "España presenta una de las tasas más altas de pobreza infantil de los países estudiados y de toda la UE: es el tercer país tanto en pobreza relativa como en 'pobreza anclada' (en ambos casos, tan solo detrás de Rumanía y Grecia). La pobreza 'anclada' habría alcanzado en España a casi el $40 \%$ de la población infantil, con un aumento de 9 puntos porcentuales entre 2008 y 2014" (en https://www.unicef.es/nota-de-prensa/la-crisis-internacional-y-laausteridadgolpean-los-ninos-en-paises-de-altos).

En todos los titulares estas personas son los objetos indirectos de la frase, pues se mencionan como sujetos pasivos, meramente receptores de la ayuda social: "Global Omnium colabora con Educo para 
incrementar el acceso de familias desfavorecidas al comedor escolar" (abc.es, 31/03/2017); "Sabadell impulsa un nuevo sistema por puntos para distribuir alimentos entre familias vulnerables" (lavanguardia.com, 19/10/2017).

A veces son cuantificados con un número que varía dependiendo de la Comunidad Autónoma, o de la ONG que actúa como fuente: "Más de 2.000 familias canarias recibieron alimentos de Cruz Roja en el último mes" (eldiario.es, 26/12/2017); "Cruz Roja ayuda a 90.000 alicantinos necesitados más que hace un año y 2.400 lactantes" (abc.es, 04/05/2017).

Sólo en 3 textos se pone rostro, e incluso nombre a los afectados por el hambre. Se trata de los escasos reportajes de interés humano que abordan el problema del hambre en España: "A veces, no comemos porque no podemos pagar ni la luz, sólo tenemos mantas para calentar a nuestros tres hijos" (eldiario.es, 15/12/2017); "Las dificultades para alimentar a mis hijas siguen siendo las mismas o parecidas" (eldiario.es, 25/05/2017); "Vamos a pasar hambre" (eldiario.es, 01/02/2017). Las ONG interpeladas sobre el tratamiento que debería darse a la pobreza infantil en España resaltaban la importancia de este tipo de contenidos, ya que "se trata de humanizar las cifras, esas que esconden tras sus números historias personales, vidas reales que pretenden acabar con la situación de precariedad que viven y a las que un tratamiento periodístico responsable puede serles de gran ayuda" (San Felipe y Mariño, 2016, p. 85).

Los 184 textos (78\%) que trabajan el tema de la solidaridad se centran en contar las diferentes iniciativas que a nivel provincial o nacional se llevan a cabo a través del Banco de Alimentos, Cáritas o Cruz Roja junto a empresas; o personas particulares encaminadas a la recogida de alimentos o recaudación de dinero para hacer frente al suministro de comida y otras necesidades básicas. Incluso los problemas a los que se enfrentan en determinadas campañas para, por ejemplo, conseguir los suficientes voluntarios -"Gran Recogida precisa 22.000 voluntarios para lograr 2,5 millones alimentos" (lavanguardia.com, 17/10/2017)- o los premios que determinadas iniciativas reciben son noticia - "Conceden a los productores de plátano el galardón de la 'Espiga de Or' de los Bancos de Alimentos" (eldiario.es, 25/10/2017)-.

Cabe destacar que el 13\% (23) de los contenidos sobre solidaridad tienen como protagonistas a multinacionales, marcas comerciales, bancos y supermercados, que, bajo el paraguas de la responsabilidad social corporativa, aportan su ayuda a la causa al mismo tiempo que se publicitan. Especialmente paradójico resulta el "Estudio sobre pobreza y alimentación", realizado por la compañía de cereales para desayuno Kellogg's con motivo del Día Mundial de la Alimentación, donde se dice que el 38\% de los encuestados quieren que su familia siga una dieta sana pero no se lo pueden permitir. Asimismo, señalan que para "el 82\% de los españoles el desayuno es la comida más importante del día" y que "el $23 \%$ de padres afirma recibir ayuda para alimentar a su familia al menos una vez al mes" (abc.es, 16/10/2017). El dietista-nutricionista Juan Revenga impulsa en Change.org una iniciativa para que cesen esas colaboraciones interesadas entre instituciones de la salud e industria alimentaria.

Esta profusión de contenidos solidarios inevitablemente coadyuva a construir la imagen de este hambre como un problema de carácter individual, donde la caridad tradicionalmente ha suplido a la responsabilidad institucional. Algunos titulares no dejan lugar a dudas en este sentido: "Parroquia Beata, Ana María Mogas: economato de pan y caridad" (abc.es, 01/04/2017); "Un total de 200 personas sin recursos degustarán en Nochebuena el Menú de Martín Berasategui para Mensajeros de la Paz" (lavanguardia.com, 13/12/2017). Si en los años 60 el director Luis García Berlanga hacía una demoledora crítica social en Plácido (1961), poniendo un pobre a la mesa de la burguesía española, 
en el siglo XXI esa imagen, sin ninguna ironía en este caso, es devuelta al imaginario social revestida de solidaridad.

Excepcionalmente, la prensa digital se cuestiona esta contradicción en algún reportaje de investigación, como "Pobreza en Canarias (IV): el derecho a la alimentación, entre el negocio y la caridad" (eldiario.es, 19/12/2017).

Sólo en un 11\% (27) de las informaciones sobre accesibilidad a nivel nacional ponen el foco de atención en las instituciones y poderes públicos. Incluso cuando esto sucede los contenidos versan sobre las restricciones y/o ayudas públicas a organizaciones sin ánimo de lucro: "El PSOE lleva al Congreso una propuesta para que el Gobierno incentive la donación de alimentos y evite su despilfarro" (lavanguardia.com, 09/03/2017). Menos frecuentes son los textos que reproducen propuestas legislativas para facilitar el acceso de la población a los alimentos: "Defensor del pueblo pide deducciones fiscales que compensen el gasto de celiacos" (lavanguardia.com, 27/04/2017); "Las ayudas a la vivienda y alimentación a personas vulnerables serán un derecho" (abc.es, 24/03/2017).

Los textos que visibilizan el "hambre local" como un problema estructural, ahondando en sus raíces y ofreciendo soluciones políticas como la renta de garantía mínima, o denunciado lo que consideran una injusticia social, son minoría, apenas un 8\% (20). En ellos se destaca la cronificación de las personas en situación de exclusión social, e incluso se alerta de "un estado que lleva aparejados problemas tanto físicos como mentales y que, a medida que pasa el tiempo, empeora" (eldiario.es, 16/02/2017). En estos casos, las fuentes son las propias ONG, quienes realizan estadísticas e informes periódicos sobre el perfil de los afectados.

La crisis económica a partir de 2008, la falta de ingresos suficientes o la precariedad laboral son algunas de las causas a las que se alude. Al mismo tiempo se constata la dificultad para cuantificar el hambre: "No hay datos oficiales sobre hambre en España, lo que hay son indicadores de pobreza. Así, dentro del embalaje del hábito de la escasez, el hambre es algo confuso que a menudo se da por hecho, pero en el que raramente se pone el foco", dice Arianna Giménez, creadora del proyecto multimedia Hungerland (elpais.com, 02/03/2017).

Las sinergias entre "hambre oculta", malnutrición y obesidad son delatadas en el artículo "Las familias con bajos recursos viven menos por la mala alimentación" (elpais.com, 17/02/2017). El aumento del precio de los alimentos saludables frente al abaratamiento de los alimentos procesados ha obligado a las familias con menos ingresos a adquirir hábitos de alimentación insanos.

Mientras abc.es se centra en los artículos sobre solidaridad-caridad, eldiario.es es el más proclive a ofrecer reportajes de interés humano, lavanguardia.com se decanta por promocionar la implicación de las empresas y sus labores solidarias, y elpais.com incluye los pocos artículos que cuestionan y profundizan sobre el "hambre oculta".

\section{Discusión y conclusiones}

La accesibilidad alimentaria, que protagoniza uno de cada diez contenidos vinculados con alimentación en los diarios digitales estudiados (elpais.com, lavanguardia.com, eldiario.es y abc.es), se representa a través de diferentes encuadres temáticos: Solidaridad (45\%), Hambre (32\%), Acceso (14\%) y Despilfarro (9\%).

La solidaridad abarca todas las iniciativas de ONG, empresas privadas y personas particulares que dedican sus esfuerzos a abastecer de alimentos fuera y dentro del espacio nacional. Son los 
contenidos más numerosos, seguidos de los que se ocupan de informar sobre el hambre. Estos se centran en la cobertura de situaciones de crisis humanitaria, sobre todo en el continente africano, aunque también en Asia y América. El diario abc.es focaliza su atención en Venezuela, lo que representa una excepción en el conjunto de los medios que merecería un estudio más pormenorizado.

Estas cifras reflejan la sobreexposición de los contenidos sobre Solidaridad, que adquieren un carácter metonímico sustituyendo las causas, origen y protagonistas del hambre por sus soluciones. El hambre que hemos denominado como "local" coincide en gran medida con la definición que la FAO ofrece del "hambre oculta". Este hambre se construye a través de los medios de comunicación como un problema que afecta a colectivos "desfavorecidos", donde las soluciones se dejan a la buena voluntad y/o la iniciativa privada; los protagonistas son las empresas, ONG y particulares altruistas, manteniendo en un segundo plano a las "víctimas" que sólo son destacadas en los titulares del 18\% de las informaciones, y en general son sujetos pasivos de unas circunstancias que parecen arbitrarias.

En su trabajo sobre las retóricas del hambre en la prensa digital española, Gracia-Arnaiz y GarcíaOliva (2017) se preguntaban si el hambre at home era real o inventada y observaban que los medios pocas veces profundizan, sobre todo cuando abordan problemas de esta complejidad. La crisis económica ha condicionado la nutrición, los hábitos alimentarios y por consiguiente la salud de los ciudadanos más desfavorecidos. Sin embargo, pocos son los datos oficiales al respecto. Algunos trabajos han intentado analizar esta situación (Atentas y Vivas, 2014; Serra-Majen y Castro-Quezada, 2014), pero estamos lejos de contar con las herramientas adecuadas para poder corroborar si ese "hambre local" está o no sobredimensionada en los medios de comunicación o si estos están haciendo dejación de sus funciones a la hora de perfilar la verdadera magnitud del problema.

Si bien coincidimos con Gracia-Arnaiz y García-Oliva (2017) en que hay un actor muy relevante en los contenidos sobre el hambre que son las entidades caritativas, humanitarias o de acción social, estas se sitúan en su estudio en tercer lugar por debajo de otros actores como las víctimas, apenas significativas en nuestra muestra, y los responsables o culpables, que, de nuevo, están casi ausentes en los textos analizados. La introducción de la pobreza y la crisis como palabras de búsqueda en su estudio y su ausencia en el presente trabajo pueden haber marcado la diferencia.

Acceso, con un 14\%, cubre varios frentes, ya que abarca todos aquellos contenidos que tienen que ver con la responsabilidad y soberanía alimentaria, pero también con los gravámenes a los alimentos, o su carestía. Por lo general son contenidos que tienen una dimensión más económica o social. Por último, Despilfarro es un encuadre que tiene un marcado tono concienciador y es el menos destacado de los publicados.

En lo que respecta a la accesibilidad alimentaria en general, lo que llama la atención es que se ha producido un desplazamiento del ámbito internacional (37\%) al nacional (52\%), quedando un 11\% para aquellas informaciones cuyo espacio de influencia o protagonismo es compartido tanto a nivel nacional como internacional.

Los 237 textos de ámbito nacional se reparten entre los siguientes encuadres: Solidaridad con un $78 \%$ (184), seguida de Despilfarro con un 11\% (26), y Acceso con un 9\% (21). Hambre es el marco que menor presencia tiene, un exiguo $2 \%$, ya que solo en 6 textos se trata de forma explícita, aunque se mencione en el titular en 11 ocasiones.

La responsabilidad institucional, las soluciones de carácter político y las causas del problema sólo aparecen mencionadas en el $8 \%$ de los textos, lo que redunda en una visión sesgada del "hambre 
local" que en muy pocas ocasiones es expuesta como un problema estructural que afecta al conjunto de la sociedad.

A este respecto, sería relevante y significativo un estudio más pormenorizado de las fuentes, sobre todo para poder verificar hasta qué punto la poca presencia de fuentes sanitarias, señalada por dichas autoras, y por el contrario la utilización de otras fuentes como ONG, políticas e institucionales influye en la construcción social del "hambre local".

Esta investigación no debería finalizar aquí, a menos que los problemas de accesibilidad, y entre ellos la llamada "hambre oculta" desaparezcan de nuestro entorno en un futuro inmediato, lo cual no dejaría de ser sino una buena noticia. Mientras eso no ocurra es necesario constatar cómo los medios de comunicación contribuyen a la construcción de este hambre y cuáles son sus límites y herramientas.

\section{Referencias}

AEDE (2016). El libro blanco de la prensa 2015 [Thewhitebook of thepress 2015]. Asociación Española de Editores de Diarios.

Atentas, J. M. y Vivas, E. (2014). Impacto de la crisis en el derecho a una alimentación sana y saludable. Informe Sepas, 2014 [Impact of the economic crisis on the right to a healthy diet. SESPAS report 2014]. Gaceta Sanitaria, 28, 58-61. http://dx.doi.org/10.1016/j.gaceta.2014.04.006

Bardin, L. (1996). Análisis de contenido [Content analysis]. Akal.

Beck, U. (2002). La sociedad del riesgo [World Risk Society]. Paidós.

Caparrós, M. (2014). El Hambre [The Hunger]. Anagrama.

Castro, S. (2013). Seguridad Alimentaria y Seguridad Global [Food Security and Global Security]. Cuadernos de Estrategia, 161, 10-23.

Clark, D. J. (2004). The production of a contemporany famine image: the image economy indigenous photographers and the case of Mechanic Philipos. Journal of International Development, 16, 693 704. http://dx.doi.org/10.1002/jid.1121

Elika (2013). Informe sobre la Seguridad Alimentaria en la prensa del País Vasco 2012 [Report on Food Safety in the newspapers of the Basque Country 2012]. https://riesgos.elika.eus/wpcontent/uploads/articulos/Archivo1086/prensa\%20completo.pdf

Elika (2014). Informe sobre la Seguridad Alimentaria en la prensa del País Vasco 2013 [Report on Food Safety in the newspapers of the Basque Country 2013]. https://riesgos.elika.eus/wpcontent/uploads/articulos/Archivo1502/SegAlimprensa2013.pdf

Elika (2015). Informe sobre la Seguridad Alimentaria en la prensa del País Vasco 2014. [Report on Food Safety in the newspapers of the Basque Country 2014]. Leioa, Universidad del País Vasco. http://www.elika.eus/datos/guias_documentos/Archivo46/Seguridad\%20alimentaria\%202014\%20 PR3\%20DIG\%20bj.pdf 
Elika (2016). Informe sobre la Seguridad Alimentaria en la prensa del País Vasco en 2015 [Report on Food Safety in the newspapers of the Basque Country 2015]. Universidad del País Vasco. https://web-argitalpena.adm.ehu.es/pasa_pdf.asp?File=USPDF164269

Entidad Catalana de Acción Social (2017). Fam oculta a Catalunya, un obstacle en la igualtat d'oportunitats http://acciosocial.org/wp-content/uploads/2017/05/FamOcultaCat informe ECASUPC 2017.pdf

Entman, R. M. (1993). Framing: toward clarification of a fractured paradigm. Journal of Communication, 43(4), 51-58. https://doi.org/10.1111/j.1460-2466.1993.tb01304.x

FAO. Seguridad alimentaria y nutricional. Conceptos Básicos [Food and nutrition security. Basic concepts]. http://www.fao.org/3/a-at772s.pdf

FAO, FIDA, OMS, PMA y UNICEF (2017). El estado de la seguridad alimentaria y la nutrición en el mundo 2017. Fomentando la resiliencia en aras de la paz y la seguridad alimentaria [The state of food security and nutrition in the world 2017. Fostering resilience for the sake of peace and food security]. FAO.

Gamson, W. A., \& Modigliani, A. (1989). Media discourse and public opinion on nuclear power: A constructionist approach. American Journal of Sociology, 95(1), 1-37.

https://doi.org/10.1086/229213

González, M. (2016). La Gastronomía como fenómeno de comunicación y relación social. Aproximaciónhistórica y estado actual [Gastronomy as a phenomenon of communication and social relationship. Historical approximation and current state]. Universidad de Vigo, Tesis doctoral.

www.investigo.biblioteca.uvigo.es/xmlui/.../la gastronomía como fenómeno.pdf.

Gracia-Arnaiz, M.; García-Oliva, M. (2017). Comiendo en tiempo de crisis: retóricas del hambre en la prensa Digital [Eating in times of crisis: rhetorics of hunger in the online newspapers]. En J.M. Comelles y E. Perdiguero-Gil (Coord.): Educación, comunicación y salud [Education, communication and health] (pp.161-181). Universitat Rovira i Virgili.

Lipovetsky, G. (2003). La era del vacio [The Empty Era]. Anagrama.

López Villafranca, P. (2012). Los encuadres sanitarios en prensa española: El caso de la gripe A y la bacteria E. Coli. [Framing in the Spanish press about the health crisis because of the E. coli bacterium]. Revista Internacional de Relaciones Públicas, 4(II), 221-246.

http://dx.doi.org/10.5783/RIRP-4-2012-10-221-246

March Cerdá, J. C. (2011). El riesgo de una mala comunicación de riesgos [The Risks of Bad Risk Communication]. Revista de Comunicación y Salud, 1(2), 61-66.

Marín Murillo, F., Armentia Vizuete, J. I. y Caminos Marcet, J. M. (2015). De lo global a lo local: el encuadre de la crisis de la carne de caballo en la prensa vasca [Global news, local coverage: how the Basque press framed the horse meat crisis]. Communication\&Society, 28(3), 29-50.

https://doi.org/10.15581/003.28.3.sp.29-50 
Mejía, L. S; Bravo, M; Mejía, S. E. (2014). La gastronomía como símbolo en la cultura [Gastronomy as a cultural icon]. Revista virtual especializada en Gastronomía, 7, 50-64.

Morais, S. (2015). Comprensión y compasión: tres décadas de representación del hambre en la prensa de referencia (El País y The New York Times) [Understanding and compassion: three decades of representation of hunger in the reference press (El Pais and The New York Times)]. Universidad Complutense. http://eprints.ucm.es/33017/1/T36347.pdf

Navas, J. (2010). El experto alimentario y los medios de comunicación: las crisis alimentarias en España (EEB 2001) frente a la tranquilidad alimentaria (2007) [The food expert and the media: food crises in Spain (BBE 2001) against food tranquility (2007)]. Athenea Digital, 18, 49-64.

Odesanya et al. (2015). Press Coverage of food Security in Nigeria: a case of The Guardian Newspaper. Journal of Economics and Sustainable Development, 6(4), 219-229.

Rodríguez, M. M. (2016). Impacto de los medios de comunicación en la cesta de la compra [Impact of mass media on the shopping cart]. Revista Española de Comunicación en Salud, 7, 69-83. http://dx.doi.org/10.7400/NCM.2014.08.2.501910.20318/recs.2016.3124

San Felipe, M. y Mariño, M. (2016). El tratamiento periodístico de la pobreza infantil según las ONG del sector: análisis y propuesta de mejora [How NGOs perceive the journalistic coverage of child poverty: analysis and suggestions]. Commons. Revista de Comunicación y Ciudadanía Digital, 5(1), 65-92. http://dx.doi.org/10.25267/COMMONS.2016.v5.i1.04

Sánchez Vera, P. (2007). Estilo de vida y cuerpo en España: un análisis en la Bourdieu [Lifestyle and body in perspectiva de Pierre Spain: ananalys is from the perspective of Pierre Bourdieu]. Pampa. Revista Interuniversitaria de Estudios Territoriales, 3(3), 219-240.

Saura, P. (2005). La gestión y la comunicación de crisis en el sector de la alimentación y bebidas [Crisis management and communication in the food and beverage sector]. Universidad Pontificia Comillas.

Sorenson, J. (1991). Mass media and discourse on famine in the Horn of Africa. Discourse\&Society, 2(2), 223-242. https://doi.org/10.1177/0957926591002002005

Serra-Majem, L. y Castro Quezada, I. (2014). La alimentación en tiempos de crisis [Food in times of crisis]. Nutrición clínica en medicina, 8(2), 35-46. http://dx.doi.org/10.7400/NCM.2014.08.2.5019

Tankard, J. W. (2001). The empirical approach to the study of Media Framing, en: S. D. Reese, O. H. Gandy y A. E. Grant, (Eds.): Framing public life. Perspectives on media and our understanding of the social world, Lawrence Erlbaum Associates: Mahwah, NJ, (pp. 95-106).

Ziegler, J. (2003). Informe del Relator Especial de la Comisión de Derechos Humanos de NNUU para el derecho a la alimentación [Report of the Special Rapporteur of the Commission on Human Rights of the UN for the right to food]. http://www.ohchr.org/Documents/Issues/Food/A.HRC.19.59.Add.5_SP.pdf 


\section{AUTORES:}

\section{Flora Marín Murillo}

Departamento de Comunicación Audiovisual y Publicidad. Universidad del País Vasco/ EuskalHerrikoUnibertsitatea (UPV/EHU). España.

En los últimos años su actividad investigadora ha estado centrada fundamentalmente en el ámbito de la alimentación y los medios de comunicación, habiendo participado en varios proyectos financiados relacionados con esta temática. En la actualidad es co-IP del proyecto financiado por el Ministerio de Economía y Competitividad sobre "Seguridad Alimentaria y cibermedios: temáticas, nuevas fuentes y servicios" (ref. CSO2017-82853-R). Con anterioridad, tomó parte del proyecto financiado por el Ministerio de Economía y Competitividad sobre "La Seguridad Alimentaria en la prensa española: estrategias para la comunicación de los riesgos alimentarios" (ref. CSO2014-54385-R).Ha sido IP del proyecto Universidad-Sociedad "La información sobre alimentación en los diarios digitales españoles: contenidos, secciones y nuevos formatos" (ref. US17/15), desarrollado en colaboración con la Fundación Eroski. También participó en un proyecto Universidad-Sociedad, cofinanciado por la ELIKA-Fundación Vasca para la Alimentación Agroalimentaria sobre "Seguridad Alimentaria y Medios de Comunicación. Un estudio de caso: la prensa vasca en 2014" (ref. US 14/13). Asimismo, en el periodo 2012-2014 participó en la realización de tres informes sobra la Seguridad alimentaria en la prensa vasca para la Fundación ELIKA. Entre 2011 y 2013 fue miembro del equipo que desarrolló el proyecto financiado por el Ministerio de Ciencia e Innovación sobre la evolución en el tratamiento de las muertes violentas por parte de la prensa vasca. Algunas de las conclusiones principales de dicho estudio han sido publicadas en el libro titulado El tratamiento de las muertes violentas en la prensa vasca (Servicio Editorial UPV/EHU, Leioa, 2012). Por lo demás, su actividad investigadora se inició hace ya 27 años con una tesis en 1992 sobre la representación de la muerte en el cine de Hitchcock. Tras participar en varios proyectos sobre el cine vasco, sus posteriores investigaciones estuvieron dirigidas al periodismo impreso y digital. Fruto de estas últimas fue la obra El diario digital (Bosch, Barcelona, 2000), uno de los primeros estudios en profundidad publicados en España sobre el que en aquel entonces se consideraba un nuevo soporte informativo. Una de las investigaciones que tuvo una especial acogida fue la realizada sobre El diario de servicios en España (Septem, Oviedo, 2002) en el que se analizaban las características de dicho modelo periodístico.

Flora.marin@ehu.eus

Índice $\mathrm{H}: 8$

Orcid ID: https://orcid.org/0000-0003-2823-598X

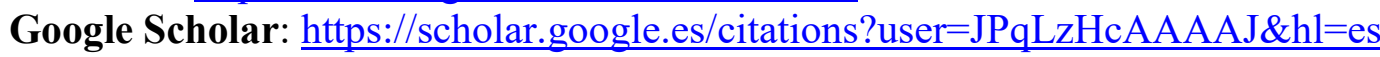

\section{José Ignacio Armentia-Vizuete}

Departamento de Periodismo. Universidad del País Vasco/ EuskalHerrikoUnibertsitatea (UPV/EHU). España.

Doctor en Ciencias de la Información, en los últimos años sus investigaciones se han centrado en el tratamiento de la Seguridad Alimentaria en los medios de comunicación. Como Investigador Principal del grupo Medialker participó en la elaboración de los Informes sobre Seguridad Alimentaria en la Prensa del País Vasco, correspondientes al periodo 2012- 2015, promovidos por Elika, la Fundación Vasca para la Seguridad Agroalimentaria. En la actualidad, es el Investigador Principal de un proyecto financiado por el Ministerio de Economía y Competitividad, titulado "Seguridad Alimentaria ycibermedios: temáticas, nuevas fuentes y servicios" (ref. CSO2017-82853R). Con anterioridad ha sido IP en otro proyecto de investigación financiado por el MINECO sobre la Seguridad Alimentaria en la prensa, y ha tomado parte en sendos proyectos Universidad-Sociedad, en colaboración, respectivamente, con Elika y con la Fundación Eroski, relacionados con estas temáticas. Durante el periodo 2011-2014 fue el Investigador Principal del proyecto de investigación 
financiado por el Ministerio de Economía y Competividad titulado "La evolución en el tratamiento de las muertes violentas en la prensa del País Vasco". Además de en diversos artículos en revistas científicas y en comunicaciones en Congresos, los resultados del proyecto se plasmaron en el libro titulado El tratamiento de las muertes violenta en la prensa vasca, publicado por la Universidad del País Vasco.En estos momentos, es el Investigador Principal del Grupo Medialker (ref. GIU16/08).

Ignacio.armentia@,ehu.es

Índice H: 17

Orcid ID: https://orcid.org/0000-0002-6570-555X

Google Scholar: https://scholar.google.es/citations?user=07ViZA8AAAAJ\&hl=es

\section{Iñigo Marauri Castillo}

Departamento de Periodismo II. Universidad del País Vasco/ EuskalHerrikoUnibertsitatea (UPV/EHU). España.

Iñigo Marauri Castillo es profesor agregado en la Facultad de Ciencias Sociales y de la Comunicación de la UPV/EHU. Tras quince años de experiencia profesional en El Correo, El País y ConsumerEroski, desarrolla su labor docente e investigadora en áreas vinculadas con la adaptación a Internet de la redacción periodística, el periodismo especializado, en particular el de sucesos y el de servicios, y la comunicación corporativa. Ha publicado más de una decena de artículos en las principales revistas de comunicación de España. Forma parte del grupo de investigación consolidado Medialker (ref. GIU16/08) y es uno de los miembros del equipo del proyecto financiado por el Ministerio de Economía y Competividad, titulado "Seguridad Alimentaria ycibermedios: temáticas, nuevas fuentes y servicios" (ref. CSO2017-82853-R). Ha participado en otros cuatro proyectos de investigación financiados en convocatorias competitivas, el último de ellos en colaboración con la Fundación Eroski, y relacionado también con la cobertura informativa de la alimentación.

Inigo.marauri@ehu.es

Índice $\mathrm{H}: 5$

Orcid ID: $\underline{\text { http://orcid.org/0000-0003-0883-8003 }}$

Google Scholar: https://scholar.google.es/citations?user=2E7JgewAAAAJ\&hl=es

\section{María del Mar Rodríguez González}

Departamento de Periodismo II. Universidad del País Vasco/ EuskalHerrikoUnibertsitatea (UPV/EHU). España.

María del Mar Rodríguez es profesora adjunta en la Facultad de Ciencias Sociales y de la Comunicación de la UPV/EHU. Sus líneas de investigación, cuyos frutos se han materializado en más de 15 artículos en las principales revistas de comunicación españolas, se han centrado en la comunicación corporativa, de manera particular en la comunicación de crisis, el periodismo de sucesos y el impacto de Internet en el periodismo de servicios, ámbito en el que ha desarrollado una trayectoria profesional durante más de 10 años como responsable de contenidos de www.consumer.es.Forma parte del grupo de investigación consolidado Medialker (ref. GIU16/08) y es uno de los miembros del equipo del proyecto financiado por el Ministerio de Economía y Competividad, titulado "Seguridad Alimentaria y cibermedios: temáticas, nuevas fuentes y servicios" (ref. CSO2017-82853-R). Ha participado en otros dos proyectos de investigación financiados en convocatorias competitivas, el último de ellos en colaboración con la Fundación Eroski, y relacionado también con la cobertura informativa de la alimentación.

mariadelmar.rodriguez@ehu.es

İndice H: 6

Orcid ID: http://orcid.org/0000-0001-9121-1468

Google Scholar: https://scholar.google.com/citations?user=KraF6dYAAAAJ\&hl=es 